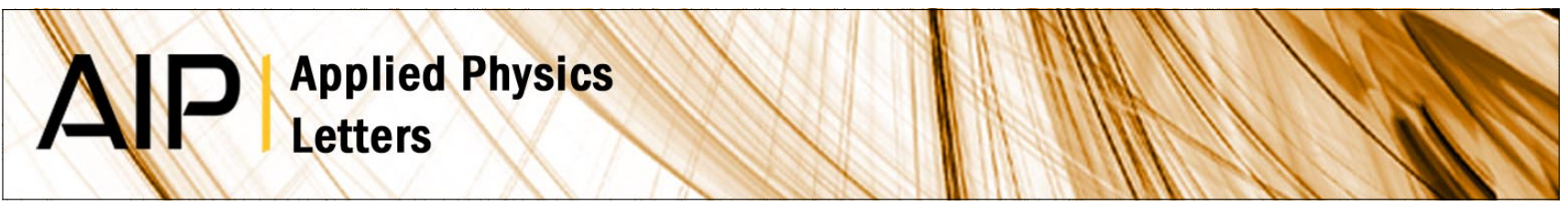

\title{
Raman scattering of folded acoustic phonons in self-assembled Si/Ge dot superlattices
}

P. H. Tan, D. Bougeard, G. Abstreiter, and K. Brunner

Citation: Appl. Phys. Lett. 84, 2632 (2004); doi: 10.1063/1.1691171

View online: http://dx.doi.org/10.1063/1.1691171

View Table of Contents: http://apl.aip.org/resource/1/APPLAB/v84/i14

Published by the American Institute of Physics.

\section{Related Articles}

All-optical tuning of the Stokes shift in $\mathrm{PbS}$ quantum dots Appl. Phys. Lett. 102, 071905 (2013)

Long wavelength $(>1.55 \mu \mathrm{m})$ room temperature emission and anomalous structural properties of $\mathrm{InAs} / \mathrm{GaAs}$ quantum dots obtained by conversion of In nanocrystals

Appl. Phys. Lett. 102, 073103 (2013)

Intraband optical transition in InGaAs/GaAs pyramidal quantum dots

J. Appl. Phys. 113, 064310 (2013)

Development of polaron-transformed explicitly correlated full configuration interaction method for investigation of quantum-confined Stark effect in GaAs quantum dots

J. Chem. Phys. 138, 054114 (2013)

Optical susceptibilities in singly charged $\mathrm{ZnO}$ colloidal quantum dots embedded in different dielectric matrices J. Appl. Phys. 113, 054303 (2013)

\section{Additional information on Appl. Phys. Lett.}

Journal Homepage: http://apl.aip.org/

Journal Information: http://apl.aip.org/about/about_the_journal

Top downloads: http://apl.aip.org/features/most_downloaded

Information for Authors: http://apl.aip.org/authors

\section{ADVERTISEMENT}

\section{AIP Applied Physics Letters}

\section{EXPLORE WHAT'S NEW IN APL}

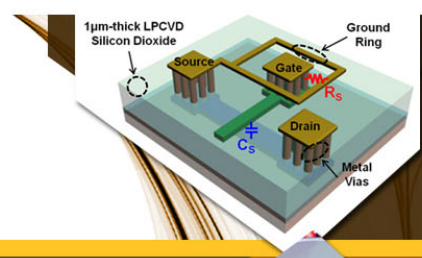

SURFACES AND INTERFACES

Focusing on physical, chemical, biological structural, optical, magnetic and electrical properties of surfaces and interfaces, and more.. 


\title{
Raman scattering of folded acoustic phonons in self-assembled Si/Ge dot superlattices
}

\author{
P. H. Tan, ${ }^{\text {a) }}$ D. Bougeard, G. Abstreiter, and K. Brunner ${ }^{\text {b) }}$ \\ Walter Schottky Institut, Technische Universität München, Am Coulombwall, D-85748 Garching, Germany
}

(Received 18 August 2003; accepted 3 February 2004)

\begin{abstract}
Self-assembled Si/Ge dot multilayers with small, uncorrelated dots fabricated by molecular beam epitaxy in the Stranski-Krastanov growth mode are studied by Raman scattering of folded longitudinal acoustic (FLA) modes. The FLA Raman spectra are analyzed and modeled with respect to mode frequencies and the spectral envelope of mode intensities. The deduced average superlattice properties are consistent with results from atomic force microscopy. The simple Rytov model used for $\mathrm{Si} / \mathrm{Ge}$ layer structures reproduces very well the frequencies of the FLA modes up to $150 \mathrm{~cm}^{-1}$. The nonlinearity of phonon dispersion curves in bulk Si for large momenta, however, becomes important for modeling the higher frequencies of observed FLA modes up to 22nd order. The effective dot layer width and an activation energy for thermal intermixing of $2.1 \pm 0.2 \mathrm{eV}$ are determined from the spectral envelopes of FLA mode intensities of as-grown and annealed $\mathrm{Si} / \mathrm{Ge}$ dot multilayers. (C) 2004 American Institute of Physics. [DOI: 10.1063/1.1691171]
\end{abstract}

Due to the lattice mismatch of $4 \%$ between $\mathrm{Ge}$ and $\mathrm{Si}$, self-organized Ge quantum dots (QDs) can be fabricated by self-assembly using the Stranski-Krastanov growth mode. ${ }^{1}$ The properties of semiconductor islands strongly depend on epitaxial deposition parameters. Self-assembled small-sized dots with a height of about two nanometers are produced by molecular beam epitaxy (MBE) at a low substrate temperature of typically $500^{\circ} \mathrm{C} .{ }^{1-3} \mathrm{Such} \mathrm{Si} / \mathrm{Ge}$ dots are very promising, for example, for light detection in the midinfrared range. ${ }^{4}$ The response of such optoelectronic structures is often increased by deposition of superlattices (SLs) with many Ge dot layers separated by $\mathrm{Si}$. Raman scattering has proven to be a versatile technique to characterize $\mathrm{Si} / \mathrm{Ge}$ layer and dot SLs. ${ }^{3,5-8}$ Folded longitudinal acoustic (FLA) modes were mainly studied in short period SLs with homogeneous layers that had to be deposited at very low substrate temperature and with surfactants in order to avoid $\mathrm{Si} / \mathrm{Ge}$ segregation and intermixing. ${ }^{5}$ Most of the Raman studies of Si/Ge dot structures were focused on their optical phonon modes and their structural properties, like Ge content. ${ }^{7,8}$ In this letter, highorder FLA phonon modes are studied in as-grown and annealed Si/Ge dot superlattices. The spectral features of acoustic Raman modes are used to characterize the structural properties of as-grown and annealed self-organized Si/Ge dot SLs.

$\mathrm{Si} / \mathrm{Ge}$ dot samples were grown in a solid source MBE system on (001)-Si substrate. A Si buffer layer with a thickness of $150 \mathrm{~nm}$ was deposited, followed by 80 periods of 8 ML Ge separated by $25.0 \mathrm{~nm}$ Si layers and a $100 \mathrm{~nm}$ Si cap layer. Several dot samples with just different amounts of boron modulation doping were grown. Only small unsystematic variations in island and Raman properties were observed. The $\mathrm{Si} / \mathrm{Ge}$ dots were formed by self-assembly at a substrate

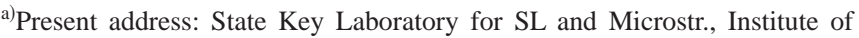
Semiconductors, Beijing, 100083, P. R. China; electronic mail: pinghengtan@hotmail.com

b) Present address: University Wuerzburg, EP 3, Am Hubland, D-97074 Wuerzburg, Germany; electronic mail: karl.brunner@physik.uniwuerzburg.de
}

temperature of $510{ }^{\circ} \mathrm{C}$. Islands in different layers are uncorrelated and not vertically stacked. Atomic force microscopy (AFM) of a reference structure with Ge islands at the surface reveals that the lateral size is about $20 \mathrm{~nm}$, the height is about $2 \mathrm{~nm}$ and the sheet density of $\mathrm{Si} / \mathrm{Ge}$ dots is about 1.5 $\times 10^{11} \mathrm{~cm}^{-2} \cdot{ }^{8}$ Raman measurements were carried out using a triple Raman spectrometer equipped with a liquid nitrogen cooled Si charge coupled device camera. An $\mathrm{Ar}^{+} 514.5 \mathrm{~nm}$ or a $\mathrm{Kr}^{+} 647 \mathrm{~nm}$ laser was used for excitation with a typical power of $1 \mathrm{~mW}$ and a spot size of $1 \mu \mathrm{m}$. The samples were in vacuum at room temperature.

Figure 1 shows the Raman spectra of the Si/Ge dot SL measured in two scattering configurations after subtraction of

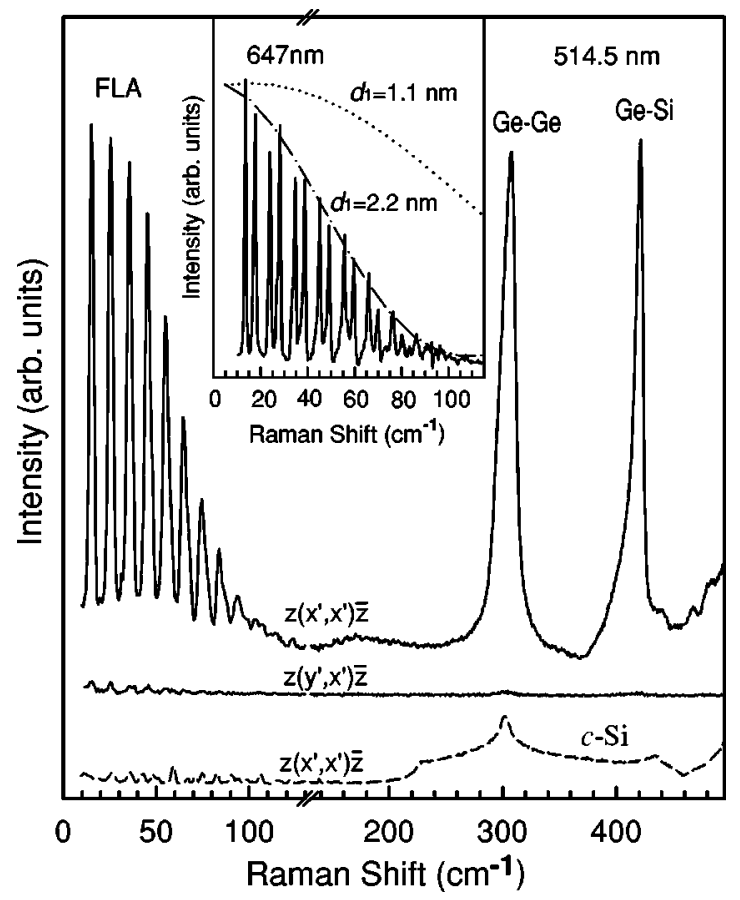

FIG. 1. Raman spectra of a Si/Ge dot SL (solid lines) and $c$-Si (dashed line) excited by a $514.5 \mathrm{~nm}$ laser. The terms $z, x^{\prime}$, and $y^{\prime}$ refer to [001], [1 $\left.1 \overline{1} 0\right]$, and [110], respectively. The inset shows the FLA Raman spectrum excited by a $647 \mathrm{~nm}$ laser. Dotted and dash-dotted lines are calculated envelopes of FLA mode intensities for dot-layer thicknesses of 1.1 and $2.2 \mathrm{~nm}$. 
the reference spectra from a Si substrate. All the Raman features originate from the $\mathrm{Si} / \mathrm{Ge}$ dots. ${ }^{8}$ The two strong peaks at high frequency are identified as Ge-Ge $\left(307.5 \mathrm{~cm}^{-1}\right)$ and Ge-Si $\left(421.5 \mathrm{~cm}^{-1}\right)$ longitudinal optical (LO) phonon modes. In the low frequency region, distinct sharp peaks up to $120 \mathrm{~cm}^{-1}$ and some weak features up to $180 \mathrm{~cm}^{-1}$ are observed. These peaks are assigned to the folded longitudinal acoustic (FLA) phonons in the $\mathrm{Si} / \mathrm{Ge}$ dot SL according to the selection rules for Raman scattering in SLs. ${ }^{5}$ The doublets of the folded phonons are not resolved here because of the small frequency splitting of less than $1 \mathrm{~cm}^{-1}$ for $514.5 \mathrm{~nm}$ excitation, which is due to the phonon wave vector being close to the SL mini zone edge. A larger doublet splitting of $4.0 \mathrm{~cm}^{-1}$ is observed up to the eighth-order FLA modes when excited by a $647 \mathrm{~nm}$ laser, as shown in the inset of Fig. 1.

The Rytov model was shown to reproduce the frequencies of the FLA modes in $\mathrm{Si} / \mathrm{Si}_{1-x} \mathrm{Ge}_{x}$ layer SLs very well, but the selection of adequate material parameters is important. ${ }^{6}$ The dot layers were described by the nominal pure $1.1 \mathrm{~nm}$ Ge layer with the sound velocity and density of bulk $\mathrm{Ge}$ or by a $2.0 \mathrm{~nm} \mathrm{Si}_{0.46} \mathrm{Ge}_{0.54}$ alloy layer with values linearly interpolated from $\mathrm{Si}$ and Ge bulk, as suggested in Ref. 6. For the Si layers we use the reduced sound velocity $\left(8.19 \times 10^{5} \mathrm{~cm} / \mathrm{s}\right)$ and density $\left(2.23 \mathrm{~g} / \mathrm{cm}^{3}\right)$ of bulk Si that were used to precisely fit the gap energy of FLA phonons in $\mathrm{Si} / \mathrm{Si}_{1-x} \mathrm{Ge}_{x} \mathrm{SLs}$ after the consideration of strain effects on the Si layers. ${ }^{6}$ The fitted result gives an average $\mathrm{Ge}$ content of $0.54 \pm 0.06$ within a $2.0 \mathrm{~nm}$ dot layer. This corresponds to an actual $\mathrm{Ge}$ content of about $0.80 \pm 0.11$ within $\mathrm{Ge} / \mathrm{Si}$ islands, if we regard islands with pyramidal shape (truncated during Si overgrowth), a height of $2 \mathrm{~nm}$ and a surface coverage of islands of $60 \%$, as analyzed by $\mathrm{AFM}^{8}$ This is in agreement with a Ge content of 0.82 within islands deduced from detailed LO phonon Raman studies. ${ }^{8}$ For both model descriptions, the calculated frequencies of the FLA mode doublets in the $\mathrm{Si} / \mathrm{Ge}$ dot SLs with a typical period of 26.3 $\mathrm{nm}$ agree very well with the experimental results in Fig. 1. The Rytov model precisely fits the frequencies of FLA modes. It should be stressed, however, that the frequencies are rather insensitive to the average composition and thickness of dot layers in the SL structures studied here, as the thickness of dot layers is less than 1/12 of that of the $\mathrm{Si}$ layers. Thus, the average sound velocity and FLA mode frequencies are mainly determined by the Si layer properties. Similarly, in x-ray diffraction (XRD) we observed SL (004) $\theta-2 \theta$ Bragg peaks up to about tenth order that can be simulated well by each of the two SL layer models. Thus, the fits of XRD curves or FLA Raman mode frequencies offer reliable values of the SL period and an average value of Ge content within a SL period. They are rather insensitive, however, to the actual composition and effective thickness of dot layers.

The Raman spectrum of a modulation-doped $\mathrm{Si} / \mathrm{Ge}$ dot SL sample in Fig. 2 shows distinct FLA modes up to 22nd order, that are presumably enhanced by resonant excitation. The highest observed mode frequency of about $220 \mathrm{~cm}^{-1}$ almost reaches the maximum value $\left(\sim 230 \mathrm{~cm}^{-1}\right)$ of the LA phonon dispersion of $c$-Ge at the edge of the Brillouin zone (BZ). The low-order FLA Raman lines are narrow and nearly

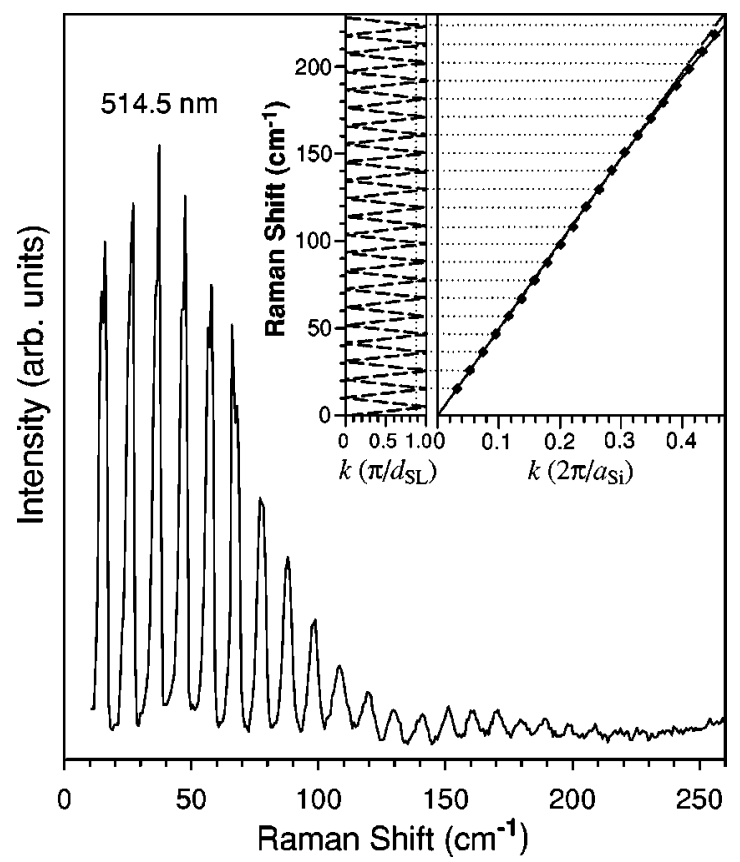

FIG. 2. FLA Raman spectra of a doped dot SL sample excited by a 514.5 $\mathrm{nm}$ laser. The insets show the calculated linear (nonlinear) dispersion curves of LA phonons as dashed (solid) lines in the SL mini zone and extended BZ schemes. The vertical dotted line in the mini zone indicates the photon momentum transfer. Diamonds show the average frequencies of observed FLA phonon doublets.

symmetric. The observation of such spectrally narrow and high-order FLA modes proves the fabrication of high-quality SLs with sharp interfaces, which was also indicated in Raman studies of optical phonons. ${ }^{8}$ The frequencies of FLA phonons are shown in the inset of Fig. 2 as diamonds in the extended BZ scheme. The calculated dispersion curves of the FLA phonons using the Rytov model are plotted in the inset of Fig. 2 as dashed lines in the mini zone and extended BZ schemes. The FLA modes in Si/Ge dot SLs up to $150 \mathrm{~cm}^{-1}$ are again well described by the Rytov model. For the FLA phonons higher than $150 \mathrm{~cm}^{-1}$, however, there exists a frequency discrepancy between the experimental data and those calculated by the Rytov model. By additional consideration of the nonlinear acoustic phonon dispersion of $c-\mathrm{Si}^{9}{ }^{9}$ the calculated dispersion curves of the $\mathrm{Si} / \mathrm{Ge}$ dot $\mathrm{SL}$ (solid line in the inset of Fig. 2) are in excellent agreement with the experimental results in the whole observed spectral range. The observed frequency reduction of the higher-order FLA modes results from the nonlinear phonon dispersion curve of $c$-Si for $k$ values above about $0.35 \times 2 \pi / a_{\mathrm{Si}}$ within the bulk $\mathrm{BZ}$.

Under nonresonant conditions fulfilled at $647 \mathrm{~nm}$ excitation, ${ }^{3}$ Raman scattering from folded acoustic phonons can be treated as a coherent sum of scattering within bulklike layers due to the photoelastic effect. ${ }^{10}$ The scattered $m$ th-order FLA Raman intensity $I_{m}$ is related to the SiGe alloy layer thickness $d_{1}$ as, ${ }^{9}$

$$
I_{m} \propto \sin ^{2}\left(m \pi d_{1} / d\right)\left[\omega_{m}\left(n_{m}+1\right)\right] / m^{2},
$$

where $\omega_{m}$ is the frequency of the $m$ th-order folded phonon, $n_{m}$ is its Bose factor, and $d=d_{1}+d_{2}$ is the SL period. We fit the spectral envelope of FLA mode intensities using the above equation (dash-dotted line in the inset of Fig. 1). The fitted thickness of the Ge dot layer is $2.2 \mathrm{~nm}$, which is in 


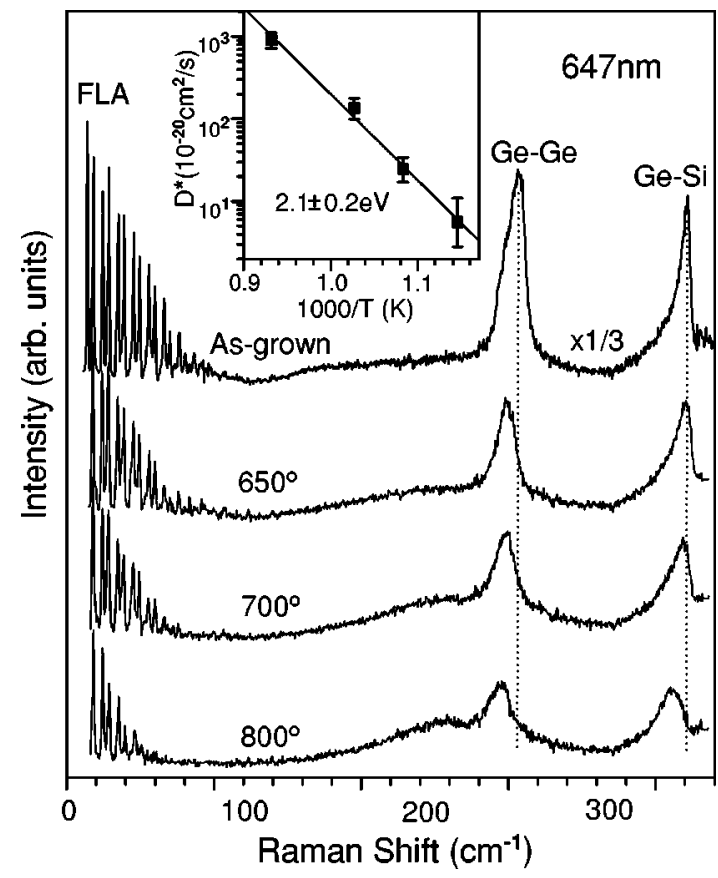

FIG. 3. Raman spectra of FLA and Ge-Ge LO phonons from an as-grown sample and from samples annealed at 650,700 and $800^{\circ} \mathrm{C}$ for $1 \mathrm{~h}$. The inset shows the deduced interdiffusion coefficient $D^{*}(T)$ vs $1 / T$.

excellent agreement with the effective height of $\mathrm{Si} / \mathrm{Ge}$ dot layers deduced from AFM studies. The calculated spectral envelope for a pure Ge layer with the nominal thickness 1.1 $\mathrm{nm}$ (dotted line in the inset of Fig. 1), in contrast, is very different from the observed profile. Therefore, the actual $(\mathrm{Si}) \mathrm{Ge}$ layer thickness that is influenced by island formation, $\mathrm{Si}$ overgrowth and possible intermixing is sensitively analyzed by the spectral envelope of FLA modes observed in nonresonant Raman studies. Island formation appears to influence the FLA Raman modes mainly by the resulting increase of the effective dot layer width.

It should be pointed out that Eq. (1) does not give a reasonable fit to the spectral envelope of the FLA mode intensities in Figs. 1 and 2 for excitation by a $514.5 \mathrm{~nm}$ laser that is close to resonance with electronic transitions of the studied $\mathrm{Si} / \mathrm{Ge}$ dots. ${ }^{3}$ Coupling between acoustic phonons and localized electronic states in $\mathrm{Si} / \mathrm{Ge}$ dots resonantly enhances Raman modes and the simple model of Eq. (1) is not valid.

Figure 3 shows the Raman spectra of an as-grown $\mathrm{Si} / \mathrm{Ge}$ dot sample and samples annealed at 650,700 , and $800^{\circ} \mathrm{C}$ for $1 \mathrm{~h}$ excited with a $647 \mathrm{~nm}$ laser. As the annealing temperature increases, the frequency of the optical $\mathrm{Ge}-\mathrm{Ge}$ and $\mathrm{Ge}-\mathrm{Si}$ modes shifts to lower frequency. The intensity of FLA phonons gradually decreases and high-order FLA modes disappear. All these features indicate that significant $\mathrm{Si} / \mathrm{Ge}$ intermixing takes place during the annealing process. ${ }^{8}$ The effective thicknesses $d_{1}$ of $\mathrm{Si} / \mathrm{Ge}$ dot layers in as-grown and annealed dot samples are obtained from their FLA mode intensity envelopes. They are summarized in Table I along with the Ge content $x_{\mathrm{Ge}}$ in the core region of $\mathrm{Si} / \mathrm{Ge}$ dots determined from the optical modes. ${ }^{8}$ The results clearly show that the higher the annealing temperature, the larger is the thickness of $\mathrm{Si} / \mathrm{Ge}$ dot layers and the smaller is $x_{\mathrm{Ge}}$. Diffusion of Si into a Ge-rich layer was shown to be much stronger than $\mathrm{Ge}$ diffusion into $\mathrm{Si}$ during annealing of $\mathrm{Si} / \mathrm{Ge}$ layer SLs. ${ }^{11,12}$ This behavior beyond Fick's law will result in a
TABLE I. Ge content $x_{\mathrm{Ge}}$ within the core and thickness $d_{1}(\mathrm{~nm})$ of Si/Ge dot layers determined from the optical and FLA modes, respectively, and deduced interdiffusion coefficient $D^{*}$ in an as-grown dot SL and those annealed at temperature $T_{A}$ for $1 \mathrm{~h}$.

\begin{tabular}{cccccc}
\hline \hline$T_{A}$ & As-grown & $600{ }^{\circ} \mathrm{C}$ & $650{ }^{\circ} \mathrm{C}$ & $700{ }^{\circ} \mathrm{C}$ & $800{ }^{\circ} \mathrm{C}$ \\
\hline$x_{\mathrm{Ge}}$ & 0.82 & 0.72 & 0.57 & 0.40 & 0.28 \\
$d_{1}(\mathrm{~nm})$ & 2.2 & 2.4 & 2.5 & 2.9 & 4.0 \\
$D^{*}\left(10^{-20} \mathrm{~cm}^{2} / \mathrm{s}\right)$ & & 5.6 & 25 & 135 & 900 \\
\hline \hline
\end{tabular}

preserved Ge-rich core with increasing thickness and decreasing $x_{\mathrm{Ge}}$, as observed for the dot SLs. We analyzed interdiffusion by regarding each layer of the flat, densely spaced islands as a homogeneous SiGe alloy layer of thickness $d_{1}$. Assuming that the observed change of $d_{1}$ represents the $\mathrm{Si} / \mathrm{Ge}$ interdiffusion length, the diffusion coefficient $D^{*}$ is given by the equation $\Delta d_{1}=d_{1}(T)-d_{1}$ (as grown) $=\sqrt{D^{*}(T) \tau}$, where $\tau$ is the annealing time. The deduced values reach $5.6 \times 10^{-20} \mathrm{~cm}^{2} / \mathrm{s}$ already at $600^{\circ} \mathrm{C}$ and describe the expansion of Ge-rich islands into the $\mathrm{Si}$ matrix. The temperature dependence is well described by an Arrhenius behavior with an activation energy of $E_{a}=2.1 \pm 0.2 \mathrm{eV}$, as shown in the inset of Fig. 3. This value is considerably lower than about $3.0 \mathrm{eV}$ (up to $4.7 \mathrm{eV}$ ) reported for Ge-rich (Si-rich) SiGe alloys. ${ }^{11,13}$ The observed enhancement of interdiffusion in $\mathrm{Si} / \mathrm{Ge}$ dot structures compared to layer structures may be attributed to strain or defects. ${ }^{13,14}$

In summary, Raman scattering of folded longitudinal acoustic phonons in $\mathrm{Si} / \mathrm{Ge}$ dot superlattices was investigated. Distinct FLA modes up to 22nd order at high frequency of $\sim 220 \mathrm{~cm}^{-1}$ are observed. The mode frequencies up to $150 \mathrm{~cm}^{-1}$ are well described by the Rytov model. The highorder FLA modes show a reduction in frequencies that is attributed to the nonlinear phonon dispersion of $c$-Si. The spectral envelopes of FLA mode intensities in as-grown and annealed $\mathrm{Si} / \mathrm{Ge}$ dot multilayers enable a sensitive analysis of effective dot layer widths and thermal interdiffusion.

The authors would like to thank I. Bormann and H. Riedl for their experimental help. This work was financially supported by the DFG(BR1960/1-3) and the BMBF(13N7870).

${ }^{1}$ Y. W. Mo, D. E. Savage, B. S. Swartzentruber, and M. G. Lagally, Phys. Rev. Lett. 65, 1020 (1990).

${ }^{2}$ K. Brunner, Rep. Prog. Phys. 65, 27 (2002).

${ }^{3}$ S. H. Kwok, P. Y. Yu, C. H. Tung, Y. H. Zhang, M. F. Li, C. S. Peng, and J. M. Zhou, Phys. Rev. B 59, 4980 (1999).

${ }^{4}$ D. Bougeard, K. Brunner, and G. Abstreiter, Physica E (Amsterdam) 16, 609 (2003).

${ }^{5}$ R. Schorer, G. Abstreiter, S. de Gironcoli, E. Molinari, H. Kibbel, and H. Presting, Phys. Rev. B 49, 5406 (1994).

${ }^{6}$ P. X. Zhang, D. Lockwood, H. Labbé, and J. Baribeau, Phys. Rev. B 46, 9881 (1992).

${ }^{7}$ A. Milekhin, N. P. Stepina, A. I. Yakimov, A. I. Nikiforov, S. Schulze, and D. R. T. Zahn, Eur. Phys. J. B 16, 355 (2000).

${ }^{8}$ P. H. Tan, K. Brunner, D. Bougeard, and G. Abstreiter, Phys. Rev. B 68, 125302 (2003).

${ }^{9}$ G. Nilsson and G. Nelin, Phys. Rev. B 6, 3777 (1972).

${ }^{10}$ C. Colvard, T. A. Gant, M. V. Klein, R. Merlin, R. Fischer, H. Morkoc, and A. C. Gossard, Phys. Rev. B 31, 2080 (1985).

${ }^{11}$ G. L. McVay and A. R. DuCharme, Phys. Rev. B 9, 627 (1974).

${ }^{12}$ R. Schorer, E. Friess, K. Eberl, and G. Abstreiter, Phys. Rev. B 44, 1772 (1991).

${ }^{13}$ S. M. Prokes and K. L. Wang, Appl. Phys. Lett. 56, 2628 (1990).

${ }^{14}$ O. G. Schmidt, U. Denker, S. Christiansen, and F. Ernst, Appl. Phys. Lett. 81, 2614 (2002). 